\title{
EVALUATION OF THE APO LIPOPROTEIN - A1 LEVELS IN TYPE II DIABETES MELLITUS, WITH AND WITHOUT CAD
}

P. V. Rajini ${ }^{1}$, A. Kasi Babu²

\section{HOW TO CITE THIS ARTICLE:}

P. V. Rajini, A. Kasi Babu. "Evaluation of the Apo Lipoprotein - A1 Levels in Type II Diabetes Mellitus, with and without CAD". Journal of Evolution of Medical and Dental Sciences 2015; Vol. 4, Issue 54, July 06; Page: 9321-9326, DOI: 10.14260/jemds/2015/1355

ABSTRACT: Coronary artery disease (CAD) had been the most common cause of death globally in diabetic patients who had been at increased risk of atherosclerosis. One of the factors contributing to coronary artery disease is the rate of altered serum concentrations of HDL-cholesterol and its apolipoprotein (apoA-1). An increase in glycosylated hemoglobin (HbA1c) greater than 8\% increases the incidence of CAD death by six times. The present study evaluated 70 patients of diabetes mellitus, with an age ranging from 40 to 60 years and with two subsets, i. e. with and without CAD. These cases had been evaluated for their serum lipid profile and apoA-1, levels along with serum creatinine and glucose. The controls were healthy individuals of age \& sex matched. The standard methodologies had been applied for analytes estimation. Apo A-1 has been measured by turbidimetric method. The study showed raised parameters of lipid profile excepting for HDL-c that was significantly lowered. Serum creatinine values showed no significance. Serum apo A-1 showed correlation with poor metabolic control, low HDL- $c$ and high triglycerides. The measurement of apo A-1 could be used as a biomarker to predict cardiovascular disease, especially in patients with type-2 diabetes mellitus.

KEYWORDS: Coronary Artery Disease = CAD; Apo lipoprotein A1=apoA-1; Diabetes mellitus = DM; HDL- cholesterol = HDL-c.

INTRODUCTION: Diabetes is now pandemic, with a worldwide incidence of $5 \%$ in general population. This situation seems to be more serious in these diabetic patients who are at increased risk of atherosclerosis and its consequences, particularly coronary artery disease (CAD). CAD had been the most common cause of death globally. Rates are higher among men than women of a given age $^{1}$. Fixed risk factors such as age, sex, family history and modifiable risk factors such as smoking, hypertension, diabetes mellitus and obesity had been well determined in this condition. One of the factors contributing to the increased risk of CAD is the high prevalence rate of lowered serum concentrations of HDL-C and its apo lipoprotein (apo A-1).2 Apo A-1 is the principal protein component of HDL, which removes cholesterol from the cells and thus having protective effect. Measurement of apo A-1, which provides further useful information, can be an alternative to the measurement of HDL-Cholesterol. The present study evaluates the atherogenic lipid profile in type 2 diabetes mellitus patients with and without coronary artery disease with focus on apo A-1.

The glycosylated hemoglobin value has been shown to predict the risk of development of many of the chronic complications in diabetes. An increase in glycoHb greater than $8 \%$, increases the incidence of death due to CAD by six times. ${ }^{3}$

Apo A-1 is the most important structural component of HDL-c. It comprises about 65 to $75 \%$ of total HDL protein. It is synthesized in the small intestine and in the liver. Apo A-1alongwith apo A II and A- IV are found on HDL. Apo A-1 binds phospholipid on lipoprotein and activates lecithincholesterol acyl transferase (LCAT), the enzyme that removes cholesterol from peripheral cells and delivers the cholesterol esters to the liver, i.e., promotes fat efflux.4,5 
MATERIALS AND METHODS: The diabetic patients were selected from the outpatient population, consulting the department of Endocrinology, Andhra medical college \& King George Hospital, Visakhapatnam. The age range was 40-60 years. No specific reference was made with regard to the sex of the patients. Coronary artery disease was defined as positive history of myocardial infarction and positive findings in the Echocardiogram of Type 2 DM patients. In the present study, cases of CAD were selected from cardiology ward, after being diagnosed by consultant cardiologist. An ethical clearance was obtained from the college ethics committee for the present study.

A total of seventy patients of both sexes were taken for the present study. A prior consent was obtained from the patients for withdrawal of blood samples. The inclusion criteria are(1) cases diagnosed with type $2 \mathrm{DM}$ and glycosylated $\mathrm{Hb}$ (HbAIc) greater than upper normal limit, indicating suboptimal glycaemic control.(2) Cases of diagnosed CAD. 30 control subjects were selected from a healthy population of no history of DM and are of age and sex matched. Exclusion criteria are those patients with hypothyroidism or trauma or with recent cerebrovascular accidents or alcoholism or the use of statins and those with chronic kidney disease.

Fasting blood samples from both subsets were obtained by venepuncture and collected in vacutainer tubes. Dry tubes were used for serum. Blood was centrifuged at $800 \mathrm{~g}$ for 15 minutes and the serum were immediately analyzed or if delayed frozen at $-20^{\circ} \mathrm{C}$ until use. The EDTA samples drawn were analyzed for glycoHb values (HbA1c).

Glycaemia was measured by glucose oxidase method 6 and HBA1c by HPLC method. ${ }^{7}$ Cholesterol (by cholesterol oxidase- PAP method); 6 HDL-cholesterol by homogenous precipitation followed by CHOD-PAP method ${ }^{6}$ and triglycerides by enzymatic method. ${ }^{6}$ ApoA-1 was measured using "Tulip" company kit according to the manufacturer's instructions. In apoA-1 estimation, in turbidimetric method, ${ }^{8}$ initial and final readings were taken at 20 and 300 seconds respectively. While interference with bilirubin, rheumatoid factor and hemolysis is avoided and samples with triglyceride levels more than $200 \mathrm{mgs} / \mathrm{dl}$ are diluted 1: 1 ratio with distilled water and measured for apo A-1 levels. Along with, serum creatinine (Jaffe's reaction) ${ }^{6}$ was also estimated.

RESULTS AND OBSERVATIONS: In the present study, 32 patients of type 2 DM with CAD and 38 patients patients without CAD had been investigated for fasting glucose, lipid profile, apoA-1 and creatinine levels. Also, glycosylated $\mathrm{HbA}_{\mathrm{IC}}$ levels were estimated. Along with, control subjects were studied.

\begin{tabular}{|c|c|c|c|}
\hline \multirow{2}{*}{ Parameter } & Controls & \multicolumn{2}{|c|}{ Test Subject with DM } \\
\cline { 3 - 4 } & Mean \pm SD & Without CAD & With CAD. \\
\hline $\begin{array}{c}\text { Serum Total Cholesterol } \\
\text { (mgs/dl) }\end{array}$ & $188.6 \pm 8.3$ & $212 \pm 12.6$ & $246.8 \pm 14.2$ \\
\hline $\begin{array}{c}\text { Serum Triglycerides } \\
\text { (mgs/dl) }\end{array}$ & $136.8 \pm 8.6$ & $220 \pm 14.8$ & $282.4 \pm 16.8$ \\
\hline Serum HDL - C (mgs/dl) & $47.6 \pm 5.2$ & $40 \pm 4.6$ & $30 \pm 3.6$ \\
\hline Serum LDL - C (mgs/dl) & $114.4 \pm 22.2$ & $128 \pm 9.6$ & $160 \pm 10.2$ \\
\hline Serum VCDL - C (mgs/dl) & $27.8 \pm 1.4$ & $44 \pm 14.2$ & $56.6 \pm 12.2$ \\
\hline HbA1c (\%) & $5.6 \pm 0.6$ & $7.8 \pm 1.2$ & $9.4 \pm 0.4$ \\
\hline Fasting Glucose (mgs/dl) & $88.6 \pm 6.8$ & $122 \pm 4.8$ & $138.2 \pm 5.6$ \\
\hline Serum Creatinine (mgs/dl) & $0.9 \pm 0.08$ & $1.12 \pm 0.3$ & $1.24 \pm 0.2$ \\
\hline \hline
\end{tabular}

Table 1: Various Parameters with mean \pm SD Values of Controls and cases (in both groups) of diabetes mellitus 


\section{ORIGINAL ARTICLE}

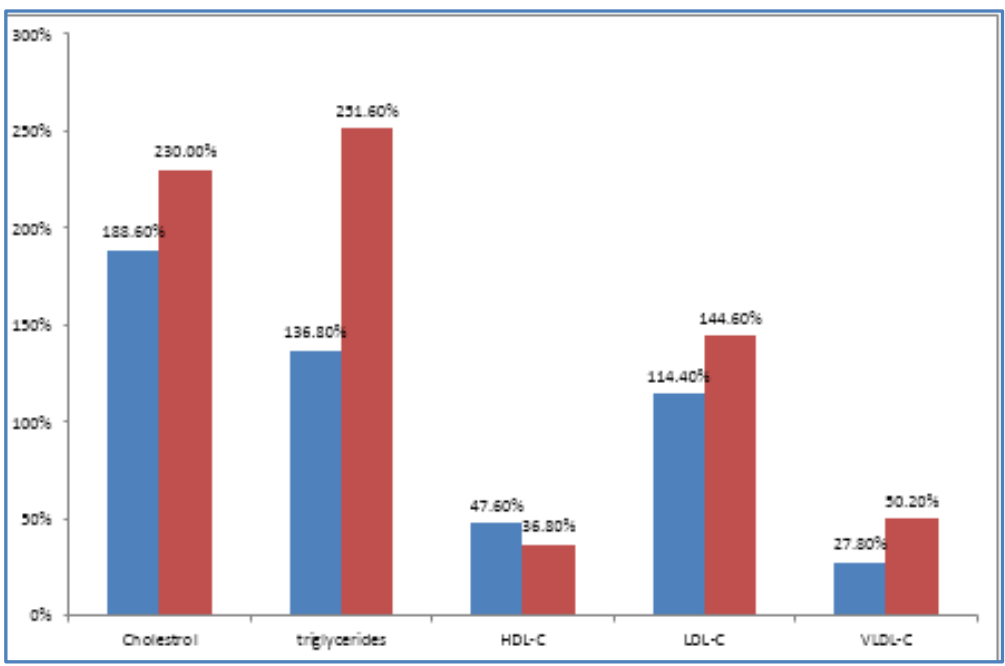

Bar Diagram 1: Mean Values of Lipid Profile In Controls And Cases

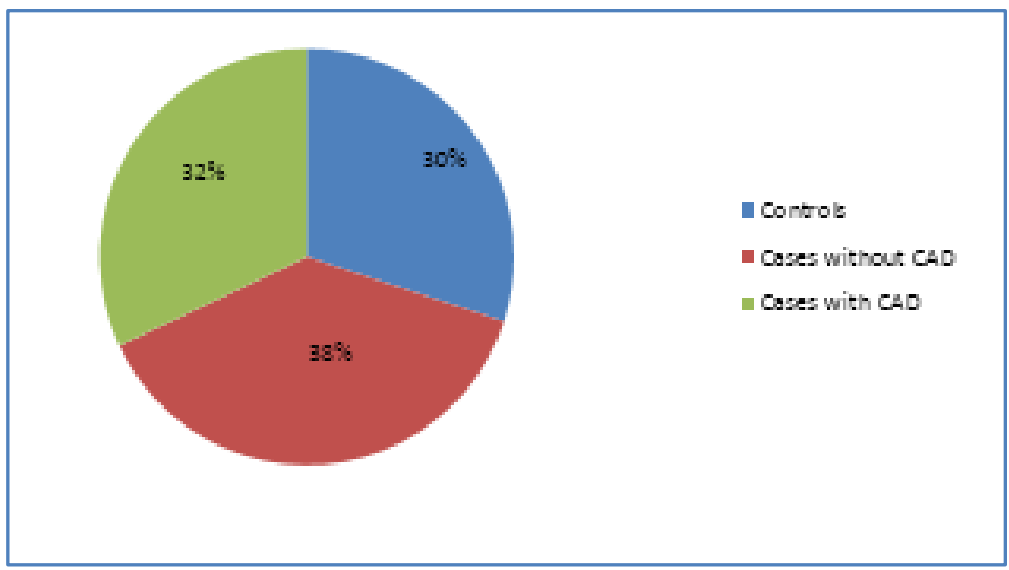

Fig. 1: Distribution of Controls and Division of Cases

The diabetic patients with CAD are of more than six years duration compared to that of cases without CAD indicating that CAD is prevalent in diabetic population of longer duration ( $>6$ years).

Apo A-1 levels are found to be lower in type 2 DM patients than those obtained in healthy individuals (P value $<0.001$ ). It is significantly decreased (Table-2).

\begin{tabular}{|c|c|c|c|c|}
\hline Variable & Mean (mgs/dl) & SD & SEM & p-Value \\
\hline APO A-1 in Cases with CAD & 90.28 & 6.14 & 1.6 & $<0.001$ \\
\hline APO A-1 in cases without CAD & 118.64 & 8.82 & 1.92 & $<0.001$ \\
\hline APO A, - Controls & 140.28 & 8.52 & 1.56 & - \\
\hline
\end{tabular}




\section{ORIGINAL ARTICLE}

Pearson correlation coefficient ( $r$ ) values were calculated for apoA-1 levels with HDL-c and triglycerides. In the present study, positive correlation was found with serum HDL-c and negative correlation with serum triglycerides. (Bar Diagram -2).

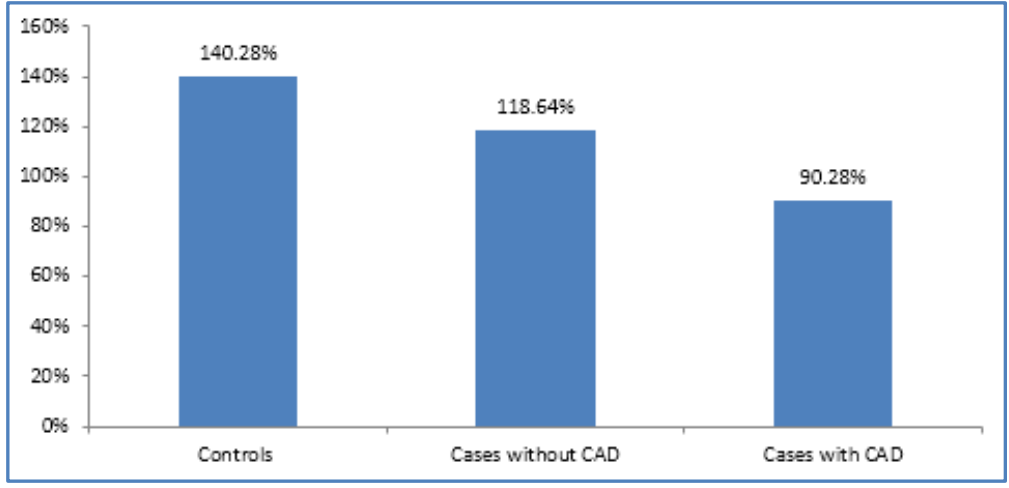

Bar Diagram-2: Mean Values of Apoa-1 in Controls and Cases with $\&$ without CAD

DISCUSSION: As per the results obtained, it is found that patients with type 2 diabetes mellitus have decreased apo A-1 levels compared to controls. The apo A-1 levels further decreased in patients with CAD, when compared to patients without CAD. ApoA-1 levels had been 90.28 46.14 Vs 140.28 \pm 8.52 in cases of CAD and controls, respectively (P Value $<0.001$ ).

It is also found that lipid profile is altered significantly in type-2 diabetic patients compared to controls. HDL cholesterol levels were significantly decreased in Type-2 diabetic patients with CAD compared to patients without CAD. Serum triglyceride levels are also found to be significantly elevated in both the groups of diabetics, compared to controls.

The study of Dean and owens ${ }^{9}$ showed a similar lowering of apo A-1 levels in diabetics. Also, Huang etal, ${ }^{10}$ in their study had observed a decrease in apo A-1 levels in diabetics with CAD. These findings had been correlated with the present study, indicating that apo A-1 might impart a protective effect from atherosclerosis. ${ }^{11}$ The possible mechanism for decreased apo-A1 in patients with type $2 \mathrm{DM}$, could be due to reduced gene expression as evident by animal models of diabetes and in cell cultures treated with high concentration of glucose. Or, it could be due to increased fractional catabolic rate of apo A-1 and HDL cholesterol as evidenced by the study of HDL kinetics by infusing leucine in patients with type 2 DM.12 In cases of diabetics with CAD, genetic factors related to apo-A1-cIII-AIV gene cluster may play a role, probably through modification of their plasma lipid pattern. And, a recent report suggests that apo A-1 mRNA is regulated by endogenously expressed antisense RNA. ${ }^{13}$ Several reports, however, indicate that apo A-1 levels are positively associated with increased risk of macro vascular disease and may represent an independent risk factor for CAD. ${ }^{14}$ Also, decreased apo A-1/HDL absolute production rate could also be the cause for incidence of CAD. ${ }^{15}$ Apo A-1 mediated cholesterol efflux ${ }^{16}$ may involve the binding of apo A-1 to the plasma membrane via its C-terminus and may require cellular ATP binding cassette transporter activity. ${ }^{17}$ Therefore, it seems that determining the number of HDL particles present in plasma or serum by measuring the concentration of apo A-1 discriminates better between patients with and without CAD than quantitating the concentration of cholsterol in these particles. Thus, apoA-1 determinations prove useful in cases of diabetics with CAD. 18,19 
In patients with type 2 diabetics, a form of dyslipidemia, that is, atherogenic profile consisting of elevated intermediate density lipoproteins, triglycerides, low density lipoproteins, very low density lipoproteins and decreased HDL ${ }^{20}$ is often observed in cases of diabetics with CAD. A study ${ }^{21}$ showed that severity of CAD is positively related to the number of triglyceride rich particles in serum which is consistent with raised triglyceride levels in the present study. Increased triglyceride concentration was associated with a reduced HDL/apo A-1 ratio suggesting that a relatively modest increase in triglycerides may rapidly alter the relative cholesterol content of HDL and thereby apo A$.22,23$ Mutation in apolipoprotein A-1 with a single amino acid replacement may form dimeric complexes and this may restrict HDL size and cause HDL deficiency. ${ }^{24}$

In the present study, higher values for HbA1c are obtained in case of poor metabolic control and persistent hypoglycemia and this might have contributed to complications such as CAD in diabetic cases.

CONCLUSIONS: The present study showed raised triglycerides levels and lowered HDL cholesterol levels in case of patients with type $2 \mathrm{DM}$, either with or without CAD, compared to control subjects. Fasting glucose and glycosylated hemoglobin (HbA1c) values are statistically higher in patients with type 2 DM with CAD-suggesting poor metabolic control in diabetic population. Serum apo A-1 showed correlation with poor metabolic control, low HDL-c and high serum triglycerides in the present study. Therefore, apart from fasting lipid profile, measurement of apo A-1 could be used as a biomarker for prediction of cardiovascular disease, especially in patients with type 2 diabetes mellitus.

\section{REFERENCES:}

1. Moran AE, Forouzamfar MH, Roth GA, Mensal GA, Ezzati M et al: "The global burden of ischaemic Heart disease in 1990 and 2010" - A study. Circulation (2010); 129 (14): 14931501.

2. Brunzell JD, Sniderman AD, albers JJ, Kuriterovich Po Jr: Apo protein B and A-1 and coronary artery disease in humans. Atherosclerosis (1984); 4 (2): 79-83.

3. Kusisto J at al: Diabetes 1994, 43, 930-937.

4. Mc queen MJ, Hawken S, Wang $\mathrm{x}$, ounpu S, Sarideman A et al: "Lipids, lipoprotein and apolipoproteins as risk markers of myocardial infarction in 52 countries"- a case control study. Lancet (2008); 372 (9634); 224-283.

5. Gugliucci A, Stahl AL; Invitroglycation of human apolipoprotein A1 reduces its efficiency in lecithin - cholesterol acyl transferase activity. Clin chim acta 1991; 204: 37-42.

6. Fundamentals of clinical chemistry - Norbelt \& Tietz $5^{\text {th }}$ edition.

7. Clinical Bio chemistry - methods and Interpretation - Harold varley, $4^{\text {th }}$ edition.

8. Methodology \& Interpretation of Biochemical Tests - Kaplan - $4^{\text {th }}$ edition.

9. Dean JD, Owens DR et al: Apo lipoprotein and lipid ratios in treated NIDDM-cardiff study. Diabetes Res 1990, 15 (1): 21-25.

10. Huang MJ and Huang B et al: Apo lipoprotien levels in normolipidemic non-insulin dependent diabetes mellitus. Diabetes care 192 (15): 1068-1074.

11. Zheng L, Nukerna B, Brennan ML, sun M, Goormastic M, settle M, et al: Apolipoprotein A-1 is a selective target for myeloperoxidase - catalysed oxidation and functional impairment in subjects with cardiovascular-disease. J clin Invest 2004, 114: 529-541. 


\section{ORIGINAL ARTICLE}

12. Drew B.G., Rye K, Duffy A, Barter S.J. \& Kingwell P.B.A. The emerging role of HDL in glucose metabolism. Nature reviews 2011; 235: 1-9.

13. Halley P, Kadakhuza BM, Faghihi MA, Magistri M, zeirer Z, khorkova O et al: "Regulation of Apolipoprotein gene cluster by a long non coding RNA". Cell Reports 2014; 6 (1) 222 - 230.

14. Francis MC, Frohlich JJ: coronary artery disease in patients at low risk apolipoprotein (APO A1) as an independent risk factor. Atherosclerosis 2001; 155: 165-170.

15. Magot et al: High density lipoprotein - apolipoprotein A-1 Kinetics in NIDDM - a stable isotope study. Diabetes care (2000 June); 23 (6): 791-795.

16. Hargrove GM, Junco A, wong NCW: Hormonal regulation of Apolipoprotein A1. J Mol endocrinol 1999; 22: $103-111$.

17. Wang N, Silver D.L, Thiele C \& Tall A. R. ATP-binding casette transporter functions as a chosterol efflux regulatory protein. J Biol chem 2001; 276: 23742-7.

18. Walladius $G$ \& Jungner I. The apo B/apo A-1 ratio: a strong new risk factor for cardiovascular disease and a target for lipid-lowering therapy: a review of evidence. J Int Med, 2006; 259: 493519.

19. Sophie Van Linhout, Frank Spillman, Heinz peter schul thiess and Carsten Tschope: High density lipoprotein at the interface of type 2 diabetes mellitus and cardiovascular disorders. Current pharmaceut design 2010; 16: 1504-1516.

20. Gotto A M Jr: Triglyceride as a risk factor for CAD. Am J cardiol 1998; 82 (22): 98 - 102.

21. Lerpian PJ, Disperse JP et al: Influence of Triglycerides concentration on the relationship between lipoprotein cholesterol and APO B and APO A-1 levels. Metabolism 1993; 42 (1): 1429-434.

22. Girard-Maudrit S. "the lipid triad or how to reduce residual cardiovascular risk". Ann Endocrinol 2010; 71(2): 89-94.

23. Andrikoula M, Mc Dowell IF. The contribution of lipid profile and apo A-1 measurements to cardiovascular risk assessment. Diabetes Obes Metab. 2008; 10(4): 271-8.

24. Sachu philip, Philips Abraham, Sheriff D.S. Apo B/Apo A-1 ratio-a better predictor of coronary artery disease in patients with or without diabetes mellitus. Int J Appl Biol Pharmaceut Tech $2011 ; 2(3): 153-158$.

\section{AUTHORS:}

1. P. V. Rajini

2. A. Kasi Babu

\section{PARTICULARS OF CONTRIBUTORS:}

1. Assistant Professor, Department of Biochemistry, AMC, Visakhapatnam.

2. Professor, Department of Biochemistry, AMC, Visakhapatnam.

FINANCIAL OR OTHER COMPETING INTERESTS: None
NAME ADDRESS EMAIL ID OF THE CORRESPONDING AUTHOR:

Dr. P. V. Rajini, Assistant Professor, Department of Biochemistry, AMC, Visakapatnam.

E-mail: rajinitallapudivsp@gmail.com

Date of Submission: 08/06/2015. Date of Peer Review: 09/06/2015. Date of Acceptance: 29/06/2015. Date of Publishing: 03/07/2015. 